Supporting Information for

\title{
Ionic environment affects bacterial lipopolysaccharide packing and function
}

\author{
Ali Rahnamoun ${ }^{\dagger}$ Kyoungtea Kim ${ }^{\ddagger}$, Joel A. Pedersen $n^{\ddagger}$, , and Rigoberto Hernandez ${ }^{\star \dagger}$ \\ †Department of Chemistry, Johns Hopkins University, Baltimore, Maryland 21218, \\ United States \\ ¥ Molecular and Environmental Toxicology Center, University of Wisconsin-Madison, \\ Madison, Wisconsin 53706, United States \\ $\S$ Departments of Soil Science, Chemistry, Civil \& Environmental Engineering, University \\ of Wisconsin-Madison, Madison, Wisconsin 53706, United States
}

This document includes the following supporting information:

- Table S1 provides several parameters for the model systems.

- Table S2 providing the parameters used in running all experiments reported in the Main document using inductively coupled plasma-mass spectrometry (ICPMS).

- Table S3 reports the mean molecular areas $\hat{A}_{\mathrm{m}}$ obtained experimentally at several different values, as indicated, of the compression modulus, $K_{\mathrm{A}}$. The largest of these values $\left(50 \mathrm{mN} \cdot \mathrm{m}^{-1}\right)$ corresponds to the transition from the LE to the liquid condensed (LC) state. An intermediate compression indicated here (20 $\left.\mathrm{mN} \cdot \mathrm{m}^{-1}\right)$ is approximately the monolayer pressure equivalent to a tensionless bilayer and exceeds that at the G-to-LE transition $\left(10 \mathrm{mN} \cdot \mathrm{m}^{-1}\right)$. The intermediate value $\left(20 \mathrm{mN} \cdot \mathrm{m}^{-1}\right)$ is also used for comparison between experiment and simulation in the Main document. The latter were performed under "tensionless" fluid lipid bilayers conditions which have been seen ${ }^{1}$ to correspond to those in the LE state of lipid monolayers at the air-water interface.

- Table S4 compares the mean molecular areas from this work to those in prior work. ${ }^{2-7}$

- Figure S1 provides a representative decay of $\hat{A}_{\mathrm{m}}$ observed in a simulation as a function of the integration time of the trajectory.

- Figure S2 illustrates how the model system was broken up into stages for the calculation of the potential between $\mathrm{OAH}$ and the bilayer using ABF.

- Figure S3 provides a comparison of the original and augmented radii in the CHARMM force field

- Figure S4 shows representative snapshots of configurations of the $\mathrm{Ca}^{2+}$ bridge in an arbitrarily selected trajectory.

- Figure S5 displays a representative snapshot of the location of OAH with respect to the LPS bilayer at which minimum free energy is observed. 
Table S1. Number of phosphoryls, Kdo sugars and charge of the deep rough LPS of Salmonella enterica. ${ }^{a}$

\begin{tabular}{cccccc}
$\begin{array}{c}\text { Phosphoryl groups } \\
\text { Per LPS }\end{array}$ & $\begin{array}{c}\text { Kdo Units } \\
\text { Per LPS }\end{array}$ & $\begin{array}{c}\text { Charge } \\
\text { Per LPS }\end{array}$ & $\begin{array}{c}\text { Total } \\
\text { Phosphoryl groups }\end{array}$ & $\begin{array}{c}\text { Total } \\
\text { Kdo units }\end{array}$ & $\begin{array}{c}\text { Total } \\
\text { Charge }\end{array}$ \\
$\mathbf{2}$ & 3 & -7 & 100 & 150 & -350 \\
\hline
\end{tabular}

${ }^{a}$ Each LPS bilayer contains 50 LPS molecules in the simulation box.

Table S2. Inductively coupled plasma-mass spectrometry (ICP-MS) instrument and acquisition parameters.

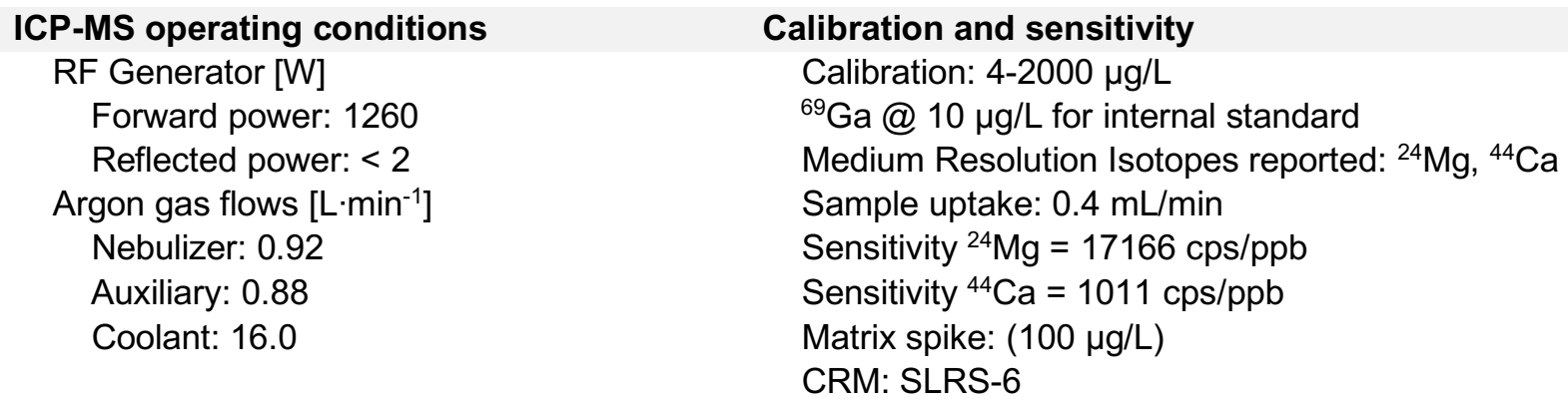

\section{Data acquisition conditions}

Dwell time: $0.010 \mathrm{~s}$

20 sample points/peak

Sweeps/reading: 4

Number of readings: 4

Mass window: $125 \%$

Peak integration window: $60 \%$

Dual counting/analog detector mode

Table S3. Mean molecular areas of interfacial LPS monolayers in the presence of alkali and alkaline earth cations obtained experimentally at the indicated compression moduli. ${ }^{a}$

\begin{tabular}{|c|c|c|c|c|c|}
\hline \multirow{2}{*}{$\begin{array}{l}\text { Added } \\
\text { Cation }\end{array}$} & \multirow[b]{2}{*}{$K_{\mathrm{A}}(\mathrm{mN} / \mathrm{m})=$} & \multicolumn{3}{|c|}{$\hat{A}_{m}\left(\AA^{2}\right)$} & \multirow[b]{2}{*}{$n$} \\
\hline & & 10 & 20 & 50 & \\
\hline none & & $233 \pm 2.6$ & $230 \pm 3.3$ & $211 \pm 4.0$ & 3 \\
\hline $\mathrm{Na}^{+}$ & & $191 \pm 1.1$ & $190 \pm 1.0$ & $174 \pm 3.3$ & 4 \\
\hline $\mathrm{K}^{+}$ & & $198 \pm 2.4$ & $195 \pm 2.5$ & $180 \pm 3.3$ & 4 \\
\hline $\mathrm{Ca}^{2+}$ & & $191 \pm 3.7$ & $190 \pm 3.4$ & $183 \pm 2.3$ & 4 \\
\hline $\mathrm{Mg}^{2+}$ & & $197 \pm 3.5$ & $198 \pm 1.3$ & $185 \pm 2.4$ & 4 \\
\hline
\end{tabular}

${ }^{a}$ Abbreviations: $\hat{A}_{\mathrm{m}}$, mean molecular area; $K_{\mathrm{A}}$, compression modulus; $n=$ number of replicates. 
Table S4. Selected experimental mean molecular areas for monolayers of lipopolysaccharides from surface pressure-area isotherms $^{a}$

\begin{tabular}{|c|c|c|c|c|c|c|c|c|}
\hline $\begin{array}{l}\text { LPS } \\
\text { type }\end{array}$ & strain $^{b}$ & cation(s) & $\begin{array}{c}\text { [cation] } \\
(\mathrm{mM})\end{array}$ & $\begin{array}{l}\text { other solution } \\
\text { constituents }\end{array}$ & $\begin{array}{c}\Pi \\
\left(\mathrm{mN} \cdot \mathrm{m}^{-1}\right)\end{array}$ & $\begin{array}{c}\hat{A}_{m} \\
\left(\AA^{2}\right)\end{array}$ & $\begin{array}{c}T \\
(\mathrm{~K})\end{array}$ & ref. \\
\hline \multirow[t]{20}{*}{$\mathrm{Re}$} & S1 & - & - & - & 20 & $182 \pm 2.3^{c}$ & 294 & this work \\
\hline & & - & - & - & 35 & $157 \pm 1.7^{c}$ & 294 & this work \\
\hline & & $\mathrm{Na}^{+}$ & 3 & - & 20 & $147 \pm 1.1^{c}$ & 294 & this work \\
\hline & & $\mathrm{Na}^{+}$ & 3 & - & 35 & $132 \pm 1.4^{c}$ & 294 & this work \\
\hline & & $\mathrm{K}^{+}$ & 3 & - & 20 & $152 \pm 1.8^{c}$ & 294 & this work \\
\hline & & $\mathrm{K}^{+}$ & 3 & - & 35 & $132 \pm 2.1^{c}$ & 294 & this work \\
\hline & & $\mathrm{Ca}^{2+}$ & 1.5 & - & 20 & $154 \pm 2.2^{c}$ & 294 & this work \\
\hline & & $\mathrm{Ca}^{2+}$ & 1.5 & - & 35 & $134 \pm 1.3^{c}$ & 294 & this work \\
\hline & & $\mathrm{Mg}^{2+}$ & 1.5 & - & 20 & $157 \pm 2.8^{c}$ & 294 & this work \\
\hline & & $\mathrm{Mg}^{2+}$ & 1.5 & - & 35 & $139 \pm 2.3^{c}$ & 294 & this work \\
\hline & & $\mathrm{Na}^{+}$ & 100 & 5 mM HEPES, pH 7.4 & 20 & $\sim 168$ & 293 & 2 \\
\hline & & $\mathrm{Na}^{+}$ & 100 & 5 mM HEPES, pH 7.4 & 35 & $\sim 134$ & 293 & 2 \\
\hline & & $\mathrm{Na}^{+} / \mathrm{Ca}^{2+}$ & $100 / 50$ & 5 mM HEPES, pH 7.4 & 20 & $\sim 138$ & 293 & 2 \\
\hline & & $\mathrm{Na}^{+} / \mathrm{Ca}^{2+}$ & $100 / 50$ & 5 mM HEPES, pH 7.4 & 35 & $\sim 126$ & 293 & 2 \\
\hline & & $\mathrm{Na}^{+}$ & 100 & 5 mM HEPES, $\mathrm{pH} 7.4$ & 20 & $\sim 168$ & 293 & 3 \\
\hline & & $\mathrm{Na}^{+} / \mathrm{Ca}^{2+}$ & $100 / 50$ & 5 mM HEPES, $\mathrm{pH} 7.4$ & 20 & $\sim 170$ & 293 & 3 \\
\hline & & - & - & $10 \mathrm{mM}$ phosphate buffer, $\mathrm{pH} 7.2$ & 30 & $\sim 120^{d, e}$ & 283 & 4 \\
\hline & & $\mathrm{Na}^{+}$ & 100 & $10 \mathrm{mM}$ phosphate buffer, $\mathrm{pH} 7.2$ & 30 & $\sim 120^{d, e}$ & 283 & 4 \\
\hline & & $\mathrm{Ca}^{2+}$ & 50 & - & 30 & $\sim 120^{d, e}$ & 283 & 4 \\
\hline & & $\mathrm{Na}^{+} / \mathrm{Ca}^{2+}$ & $100 / 50$ & - & 30 & $\sim 120^{d, e}$ & 283 & 4 \\
\hline \multirow[t]{2}{*}{ Rc } & E1 & $\mathrm{Na}^{+}$ & $20 ?$ & $20 \mathrm{mM}$ sodium phosphate, $\mathrm{pH} 7.0$ & 20 & $\sim 107$ & $\mathrm{RT}^{f}$ & 5 \\
\hline & & $\mathrm{Na}^{+}$ & $20 ?$ & $20 \mathrm{mM}$ sodium phosphate, $\mathrm{pH} 7.0$ & 35 & $\sim 92$ & $\mathrm{RT}^{f}$ & 5 \\
\hline \multirow[t]{6}{*}{$\mathrm{Ra}$} & S2 & $\mathrm{Na}^{+}$ & 100 & 5 mM HEPES, pH 7.4 & 20 & $\sim 205$ & 293 & 2 \\
\hline & & $\mathrm{Na}^{+}$ & 100 & 5 mM HEPES, pH 7.4 & 35 & $\sim 180$ & 293 & 2 \\
\hline & & $\mathrm{Na}^{+} / \mathrm{Ca}^{2+}$ & $100 / 50$ & 5 mM HEPES, pH 7.4 & 20 & $\sim 164$ & 293 & 2 \\
\hline & & $\mathrm{Na}^{+} / \mathrm{Ca}^{2+}$ & $100 / 50$ & 5 mM HEPES, pH 7.4 & 35 & $\sim 138$ & 293 & 2 \\
\hline & E2 & $\mathrm{Na}^{+}$ & 150 & 10 mM HEPES, pH 7.4 & 35 & $188 \pm 1.3$ & 294 & 6 \\
\hline & & $\mathrm{Na}^{+} / \mathrm{Ca}^{2+}$ & $150 / 50$ & 1 mM EDTA, 10 mM HEPES, $\mathrm{pH} 7.4$ & 35 & $156 \pm 3.2$ & 294 & 6 \\
\hline \multirow[t]{2}{*}{ wild type } & E3 & $\mathrm{Na}^{+}$ & 100 & $10 \mathrm{mM}$ HEPES, $\mathrm{pH} 7.4$ & 15 & $\sim 300^{e}$ & $\mathrm{RT}^{f}$ & 7 \\
\hline & & $\mathrm{Na}^{+} / \mathrm{Ca}^{2+}$ & $100 / 20$ & 10 mM HEPES, $\mathrm{pH} 7.4$ & 15 & $\sim 250^{e}$ & $\mathrm{RT}^{f}$ & 7 \\
\hline
\end{tabular}

${ }^{a}$ Abbreviations: $\hat{A}_{\mathrm{m}}$, mean molecular area; RT, room temperature; $T$, temperature; $\Pi$, monolayer lateral pressure. ${ }^{b}$ Bacterial strains: $\mathrm{E} 1$, Escherichia coli J5; E2, E. coli K-12; E3, E. coli O55:B5; S1, Salmonella enterica serovar minnesota R595; S2, S. enterica serovar minnesota R60. ${ }^{c}$ Purified salts. ${ }^{d}$ LPS in native

salt form. ${ }^{e}$ Salts used as received. ${ }^{f}$ Assumed. 


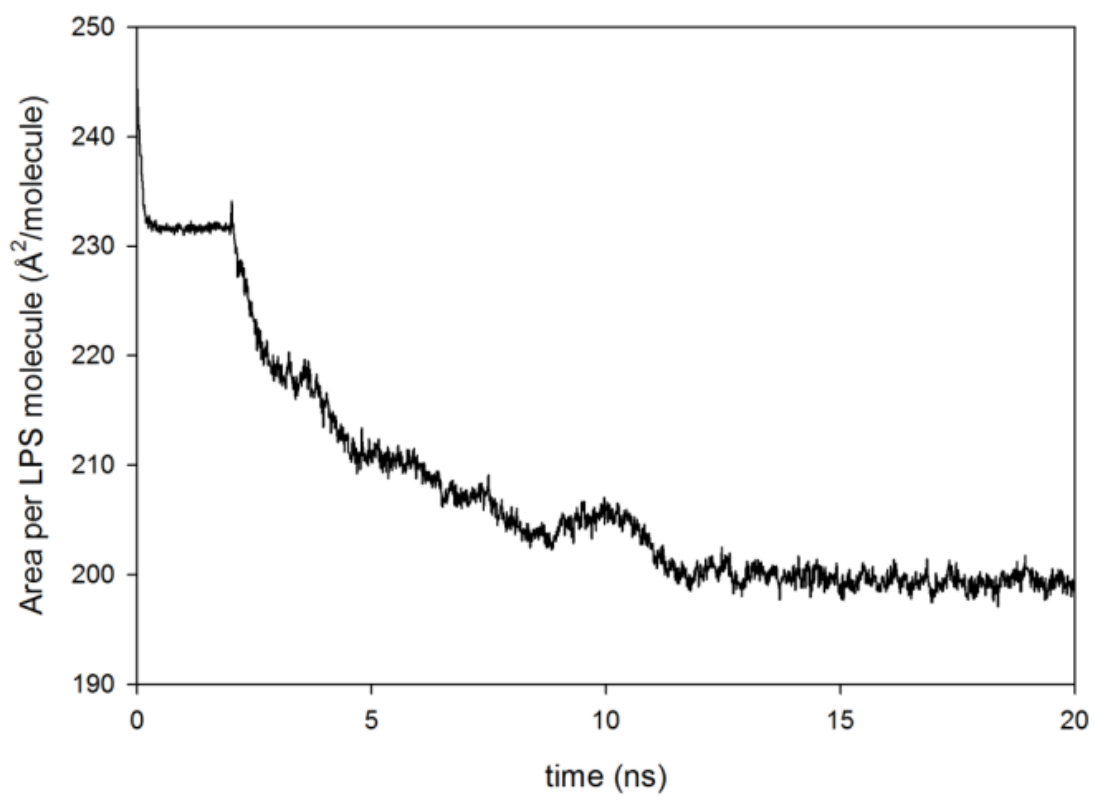

Figure S1. Evolution of the effective mean molecular area $\left(\hat{A}_{m}\right)$ molecule during a single trajectory equilibration of deep rough Salmonella enterica in $\mathrm{Ca}^{2+}$ ionic environment.

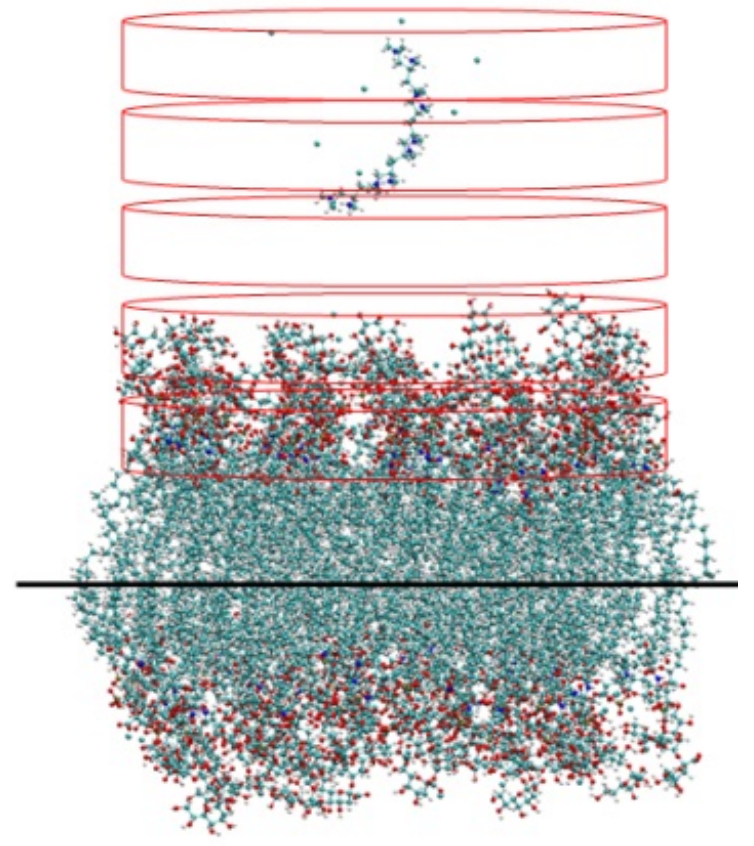

Figure S2. ABF sampling while the COM distance between $\mathrm{OAH}$ and the carbon atoms of the acyl chain terminal methyl group of the lipid A sections of the LPS molecules is changing from $50 \AA$ to $10 \AA$. The sampling areas in separate ABF calculations are shown with red cylindrical shapes.Implicit water solvent molecules are not shown for clarity. 


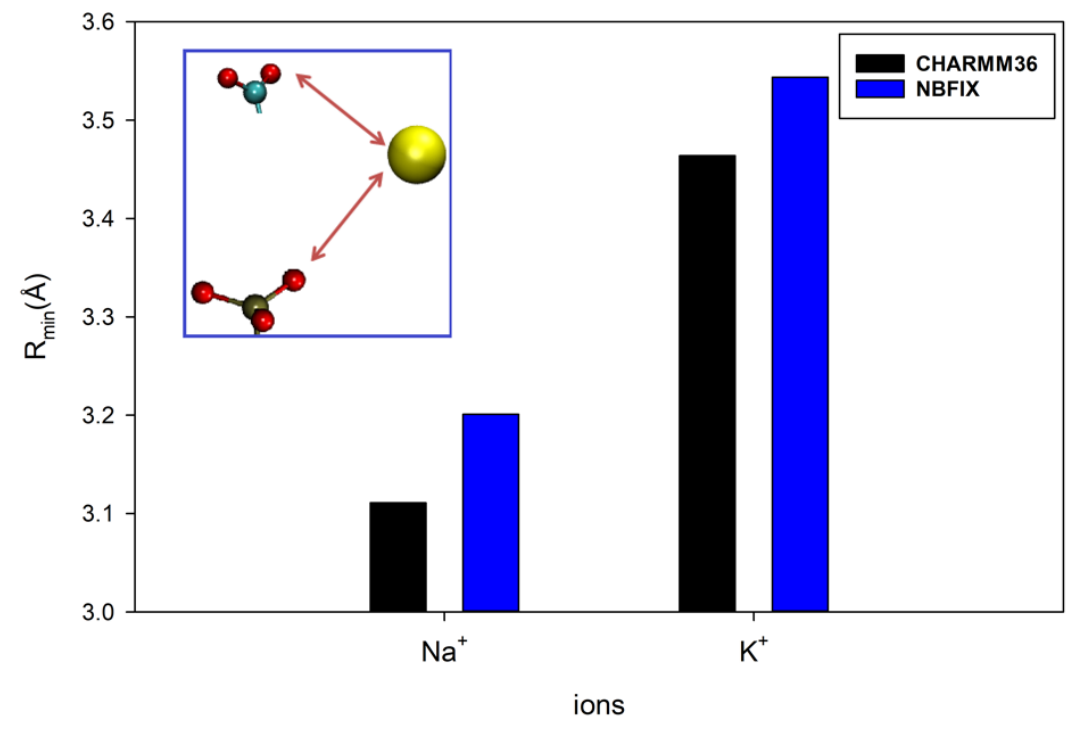

Figure S3. Original and augmented CHARMM LJ radii for the interactions between monovalent cations and oxygen atoms of carboxyl and phosphoryl groups. Pair-specific non-bonded interactions between monovalent cations and oxygen atoms of carboxyl and phosphoryl groups. LJ radii of these pair interactions were calibrated against experimentally measured quantities.

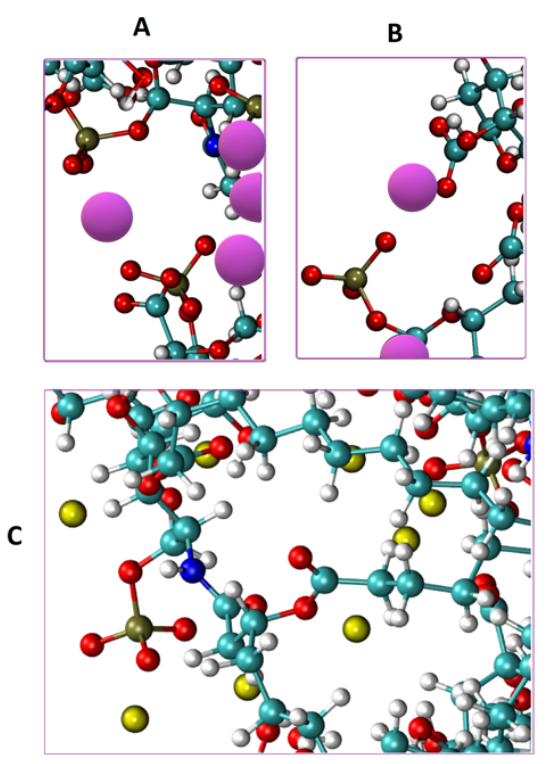

Figure S4. Sample snapshots of a $\mathrm{Ca}^{2+}$ bridge between (A) phosphoryl-phosphoryl and (B) carboxyl-phosphoryl groups in the core region of deep rough LPS from Salmonella enterica. Carbon atoms are shown in cyan, Oxygen atoms are shown in red, Hydrogen atoms are shown in white and Phosphorous atoms are shown in dark gold. The $\mathrm{Ca}^{2+}$ ions are shown in purple. (C) Sample snapshot of charge shielding of anionic groups by $\mathrm{K}^{+}$monovalent cation. $\mathrm{Na}^{+}$ions are shown in yellow. 


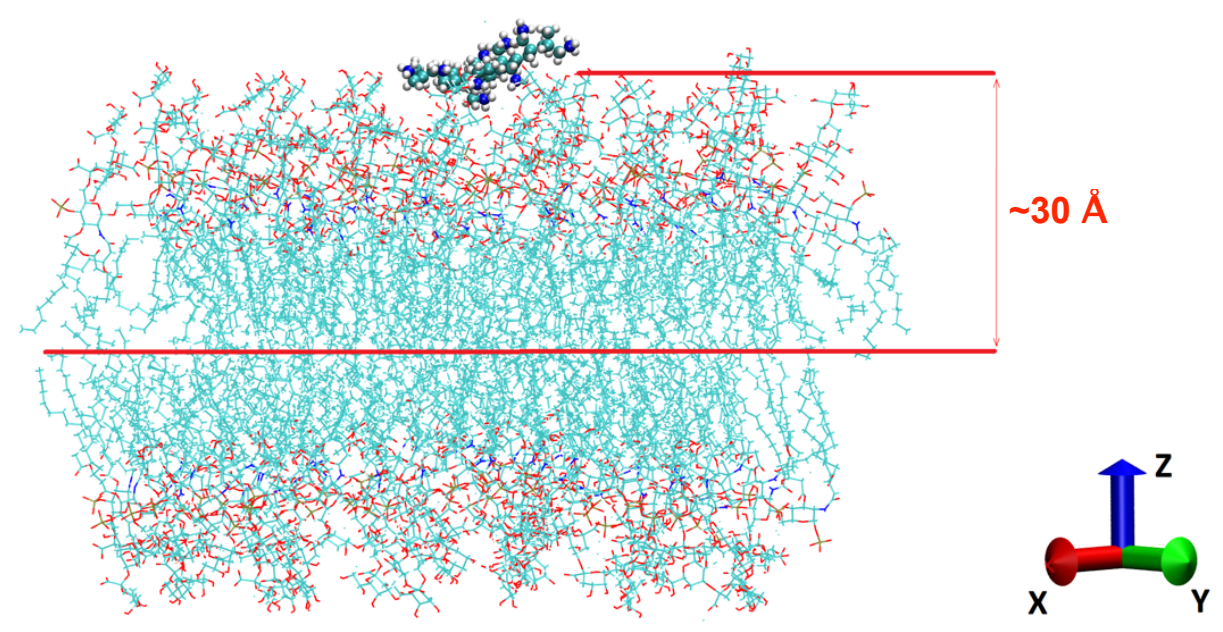

Figure S5. Snapshot of the location of OAH with respect to the LPS bilayer at which minimum free energy is observed.

\section{References}

1. Marsh, D. Lateral pressure in membranes. Biophys. Biochim. Acta 1996, 1286, 183-223.

2. Herrmann, M.; Schneck, E.; Gutsmann, T.; Brandenburg, K.; Tanaka, M. Bacterial lipopolysaccharides form physically cross-linked, two-dimensional gels in the presence of divalent cations. Soft Matter 2015, 11, 6037-6044.

3. Oliveira, R. G.; Schneck, E.; Quinn, B. E.; Konovalov, O. V.; Brandenburg, K.; Gutsmann, T.; Gill, T.; Hanna, C. B.; Pink, D. A.; Tanaka, M. Crucial roles of charged saccharide moieties in survival of gram negative bacteria against protamine revealed by combination of grazing incidence $\mathrm{x}$-ray structural characterizations and Monte Carlo simulations. Phys. Rev. E 2010, 81. 4. Jeworrek, C.; Evers, F.; Howe, J.; Brandenburg, K.; Tolan, M.; Winter, R. Effects of specific versus nonspecific ionic interactions on the structure and lateral organization of lipopolysaccharides. Biophys. J. 2011, 2169-77.

5. Le Brun, A. P.; Clifton, L. A.; Halbert, C. E.; Lin, B.; Meron, M.; Holden, P. J.; Lakey, J. H.; Holt, S. A. Structural characterization of a model Gram-negative bacterial surface using lipopolysaccharides from rough strains of Escherichia coli. Biomacromolecules 2013, 14, 20142022.

6. Hwang, H.; Paracini, N.; Parks, J. M.; Lakey, J. H.; Gumbart, J. C. Distribution of mechanical stress in the Escherichia coli cell envelope. Biochim. Biophys. Acta 2018, 1860, 25662575.

7. Micciulla, S.; Gerelli, Y.; Schneck, E. Structure and conformation of wild-type bacterial lipopolysaccharide layers at air-water interfaces. Biophys. J 2019, 116, 1259-1269. 